\title{
EL BACHILLERATO UNIVERSITARIO Y LA REFORMA DEL NIVEL MEDIO SUPERIOR, POTENCIALIDADES PARA LA VINCULACIÓN
}

\begin{abstract}
Jesús Ruiz Flores
La creación en este sexenio de una Subsecretaría de Educación Media Superior, además de que pudiera considerarse como una señal de la importancia que pretende el gobierno asignar a este nivel, ofrece posibilidades de vincular más productivamente las acciones de política pública con las acciones de las instituciones educativas y éstas, a su vez, con las acciones que es preciso implementar, a nivel de la sociedad en su conjunto, para lograr avances en el país. Al parecer hay voluntad y decisión de todos los sectores sociales para destinar cada vez mayores recursos al nivel. Hay un proceso de reforma nacional del bachillerato, que se orienta por un modelo de competencias, situación de la cual, el bachillerato de la Universidad de Guadalajara no puede estar al margen. El presente trabajo es una reflexión a partir de una búsqueda de la identidad de la vinculación en el nivel medio superior de la Universidad de Guadalajara, nivel en el cual ofrece tanto el bachillerato general como el técnico y carreras técnicas. La actividad desde la que se reflexiona se orientó a rescatar experiencias de las diferentes escuelas que conforman el Sistema de Educación Media Superior y buscar potenciarlas hacia formas de vinculación novedosas.
\end{abstract}

Palabras Clave: Educación media superior. Reforma del bachillerato. Vinculación.
Profesor Investigador; Centro Universitario de la Ciénega Universidad de Guadalajara. México.

mgems.ruiz@gmail.com 


\section{$1 \quad$ Introducción}

Hoy en día se habla con insistencia de la necesidad de reformar la educación media superior, partiendo de visiones en las que se identifica que la situación que guarda el nivel no es necesariamente la más deseable. En ese sentido, se habla de las crisis y conflictos de ese nivel de estudios, tanto por parte de organismos internacionales, sectores empresariales, así como de investigadores educativos y cuerpos académicos especializados en el nivel.

La creación en este sexenio de una Subsecretaría de Educación Media Superior, además de que pudiera considerarse como una seńal de la importancia que pretende el gobierno asignar a este nivel, ofrece posibilidades de vincular más productivamente las acciones de política pública con las acciones de las instituciones educativas y éstas, a su vez, con las acciones que es preciso implementar, a nivel de la sociedad en su conjunto, para lograr avances en el país.

Al parecer hay voluntad y decisión de todos los sectores sociales para destinar cada vez mayores recursos al nivel, se impulsa una reforma nacional del bachillerato, situación a la cual, el bachillerato de la Universidad de Guadalajara (U de $\mathrm{G}$ ) no puede estar exenta.

El presente documento explora las potencialidades que representa el bachillerato universitario -siendo el caso de la Universidad de Guadalajara, que al tiempo de ofrecer educación superior tiene también educación media superior -, en un contexto en que el nivel medio superior a nivel nacional está en un proceso de reforma, en tanto que la Universidad de Guadalajara está arrancando una reforma curricular basada en la formación por competencias.

\section{La Educación Media Superior en el PND}

De acuerdo con el Plan Nacional de Desarrollo (PND), para el período 2007-2012, el eje fundamental en torno al cual giran las estrategias del 
gobierno federal en el rubro de educación es: "elevar la calidad educativa". Esta aspiración ha estado presente a lo largo de los más recientes gobiernos, sin embargo, para los distintos actores sociales existían dudas en términos de qué era lo que debería entenderse por este concepto. En el afán de subsanar la indefinición que a lo largo del tiempo ha estado presente, el documento señala que el concepto de calidad está conformado por los criterios de "cobertura, equidad, eficacia, eficiencia y pertinencia."

Por lo tanto, podemos afirmar que estos son los principios de los cuales se desprenden los objetivos, las estrategias y las políticas propuestas por el gobierno en materia educativa.

Para el caso de la educación media superior, se proyecta como objetivo principal, "[...] fortalecer el acceso y la permanencia en el sistema de enseñanza media superior, brindando una educación de calidad orientada al desarrollo de competencias". (PND, 2007, p. 190).

\section{Situación del nivel}

En la actualidad hay en el país un total de 13,521 escuelas de educación media superior, de las cuales 5,460 son privadas y el resto son planteles públicos. Están inscritos en este sector educativo, 3 millones 855 mil estudiantes, que son atendidos por $266 \mathrm{mil}$ profesores. Es decir, de acuerdo con estos datos que corresponden al ańo 2007, en promedio hay I 4.5 alumnos por cada profesor. Sin embargo, habría que preguntarse si esta relación numérica maestroalumno, es igual para el control público como para el privado.

El total de alumnos inscritos en este nivel, además de que se reconoce como uno de los niveles que presentará un fuerte crecimiento en su matrícula en los próximos años, es sumamente importante, ya que el dato, por si mismo representa un número mayor a la población que tienen más de medio centenar de países en el mundo. 
No obstante, de acuerdo con los datos que reporta la Organización para la Cooperación y el Desarrollo Económico (OCDE), en México hay 30.2 estudiantes por profesor en las escuelas públicas, y i 6.6 en las privadas. El promedio de los países de la OCDE para este mismo indicador es I3.2, para las públicas, y I2 para las privadas. Esto significa que los grupos de estudiantes de educación media superior pública son un poco más del doble que los del promedio de la OCDE y que el promedio de alumnos de escuelas particulares apenas es más alto que dicho promedio.

En lo que respecta a la cobertura de educación media superior, a pesar del crecimiento en la matrícula, sólo se logra atender el 58\% de los jóvenes que están en edad de estudiar este nivel, siendo que en los países de la OCDE este indicador es mayor al $80 \%$.

Otro dato que refleja este rezago en la cobertura, es el porcentaje de egresados de educación media superior, con respecto a la población que está en edad típica de graduación. En este indicador México se encuentra en último lugar del conjunto de países de la OCDE, con $40 \%$, mientras que el promedio es $82 \%$. Incluso, países como Chile y Brasil reportan una tasa de graduación para el nivel de $73 \%$.

\section{$4 \quad$ El Bachillerato en Jalisco}

En nuestro país la educación obligatoria comprende I2 años, tres de preescolar (a pesar de que en 2008 la Secretaría de Educación Pública [SEP] reconoció importantes y severos problemas para alcanzar la cobertura en los tres grados), seis de primaria y tres de secundaria. No obstante, en algunas legislaciones estatales se establecen is años de educación obligatoria. Es el caso del estado de Jalisco en donde la educación media superior está considerada en este carácter de obligatoriedad. 
Sin embargo, la situación actual no corresponde con este mandato legal. Por ejemplo, mientras la cobertura es de $58.6 \%$, en el Distrito Federal es de 90.1\%; en otros estados como Morelos y Sinaloa alcanzan alrededor de $67 \%$, en Jalisco se reporta sólo 51.3\%, lo cual sitúa a nuestra entidad en el lugar vigésimo cuarto del conjunto de los 32 estados de la república. Atender de manera prioritaria y fundamental la llamada educación obligatoria no es una cuestión que se logre mediante un decreto, sino con voluntad política y con una serie de medidas integrales de atención a todos los niveles. El caso de la obligatoriedad de la educación preescolar en nuestro país, es el ejemplo más ilustrativo de ello.

Con respecto a la absorción en bachillerato de alumnos egresados de la secundaria, el indicador para Jalisco es de $80.6 \%$, mientras que en el Distrito Federal alcanza el 107.9\% (esta cifra se explica en virtud de que muchos egresados de la zona metropolitana, pertenecientes al Estado de México, solicitan su inscripción en el D.F.) y en estados como Sinaloa es de 94.8\%. En este rubro, nuestra entidad se ubica apenas en el lugar 26.

De acuerdo con las cifras de la Secretaría de Educación Pública (SEP), en el período 2005-2006, en el Estado de Jalisco había 212,763 alumnos en educación media superior. De estos 48,954 eran atendidos por escuelas privadas, lo cual representaba $23 \%$ del total. Destaca que, de 1990 a la fecha, la matrícula privada de educación media superior ha pasado de representar sólo el ro\% hasta el $23 \%$ actual. Es decir, que el crecimiento del sector privado se ha incrementado de manera notable en los últimos ańos, pasando de 17 mil estudiantes que había en 1990, a casi el triple, con los 49 mil que se reportan en 2005.

Otro dato a considerar es que la U de G atiende al 50\% del total de alumnos que están estudiando algún programa de educación media superior en Jalisco. Es decir, que en este nivel educativo la presencia de la Ude es bastante significativa. Esto es, del total de la matrícula de la U de G, el $58 \%$ corresponde a alumnos de educación media superior. Por tal motivo, la U de G y la Universidad Nacional Autónoma de México (UNAM) son 
las universidades del país que atiende a un mayor número de estudiantes de este nivel, ambas con una matrícula de poco más de Ioo mil alumnos en cada una de ellas.

\section{La vinculación en el bachillerato de la Universidad de Guadalajara}

El bachillerato en la Universidad de Guadalajara está en proceso de reforma y cuenta, cada vez más y de manera creciente, con una planta académica más profesionalizada, capacitada; cada día más dispuesta a trabajar en proyectos de mejora institucional.

Uno de los esfuerzos que permiten hacer este tipo de aseveraciones es la realización de diversos foros para revisar las funciones sustantivas del nivel. Uno de ellos es el realizado en noviembre de 2008, desde la Dirección de Educación Técnica del Sistema de Educación Media Superior (SEMS) de la Universidad de Guadalajara, que cubrió el propósito de acercarse al concepto vinculación, las actividades que este rubro comprendían y las condiciones en que se desarrollan en las escuelas con educación técnica del SEMS.

Estas líneas no representan en mucho una visión completa de las condiciones y productos de la vinculación en las escuelas con educación técnica, pues ha hecho falta continuidad en los trabajos para recuperar e integrar la información. Se ofrece en cambio una visión de conjunto que apunta en todo caso a una probable categorización que, más que en sentido valorativo se hace resaltando por una parte la antigüedad que tiene las carreras como oferta educativa y por otro lado su liga a determinados mercados de trabajo.

Se agrega como corolario a esta descripción, la definición más comúnmente aceptada del concepto por parte de los coordinadores, así como las deficiencias señaladas y demandas expresadas. Por último, una reflexión del autor de estas líneas. 


\section{Una visión de conjunto de las formas de vinculación}

Entre las actividades de vinculación se encuentran el "servicio social y las prácticas profesionales"; de forma relativamente reciente y sistemática se hace "seguimiento de egresados" a través de la aplicación de la ficha preegreso, así como un tanto más del seguimiento de egresados propiamente dicho. Se incluye también "el impulso a la titulación", del cual se han producido algunas evaluaciones que indican una mejora en este rubro.

En algunas escuelas se llega a manejar una "bolsa de trabajo" para los egresados, en buena parte debido a la relación que diversos docentes tienen con empresas en las que laboran o han laborado y por las relaciones informales con los directivos de las empresas.

La responsabilidad por el diseño, operación, seguimiento y evaluación de los programas de servicio social y prácticas profesionales se halla normado en el Estatuto Orgánico del Sistema de Educación Media Superior, mas existen distancias entre la norma y las posibilidades operativas que los coordinadores llegaron a señalar en el foro y que mencionaremos más adelante.

Para el seguimiento de egresados, en 1998 se empezaron a sentar bases organizativas y financieras para la creación de Unidades de Vinculación -a la par que en cada uno de los centros universitarios- en cada una de las escuelas con oferta de educación técnica, pero por diversos motivos no llegaron a concretarse; sin embargo, como función, el seguimiento de egresados se hizo en un solo ejercicio por parte de las escuelas, coordinados por la administración central del SEMS. Posteriormente, hacia 200I-2005, desde la misma administración central del SEMS se contrataron varios levantamientos al Centro de Estudios de Opinión de la propia Universidad.

Dichos estudios no se aplicaron orientados a generar diagnósticos y análisis de requerimientos sociales relacionados con cada área profesional para la actualización e innovación de los programas educativos. 
Algunas escuelas han llegado a experimentar una modalidad de "estancia laboral" así como las "cátedras empresariales", pero son casos muy aislados. A nivel universidad, al parecer promovidas por la Fundación Universidad de Guadalajara, se han presentado cátedras con figuras ampliamente conocidas y reconocidas de organizaciones empresariales o de empresas específicas.

Igualmente se han llegado a presentar "donación de equipo o habilitación de laboratorios" a cambio de "desarrollo de prototipos", pero sólo de manera muy incipiente y básica. Aquí se puede hablar de una extendida deficiencia en el equipamiento de talleres y laboratorios, que bien se puede ayudar a superar con el apoyo a ideas que se empiezan a generar en algunas escuelas.

Las "expos" organizadas por las propias escuelas - en las que las preparatorias no han estado al margen -, así como la participación en las organizadas por instituciones diversas a la $\mathrm{UdeG}$, son otra estrategia que se implementa en diversas carreras y escuelas.

Una novedad en el Sistema es el acuerdo del SEMS con la Cámara Nacional de la Industria Electrónica, Telecomunicaciones e Informática (CANIETI) en 2007 y el concurso "Universitrónica”, que por cierto fue la primera ocasión que se dio en un espacio universitario. Estos eventos convoca a equipos de estudiantes del área de la electrónica a participar en un concurso de proyectos novedosos y a los industriales e inversionistas, que acuden en busca de proyectos prácticos para financiar el desarrollo y comercialización, la selección de aquellos que les parecen prometedores y dar pie a la creación de nuevos negocios, donde los autores de los proyectos quedan como socios mayoritarios.

Se puede observar que tienen poca presencia otras formas de vinculación, como son los estudios de trayectorias académicas y laborales, que ayudarían a entender la compleja relación entre la educación, sociedad y mercados de trabajo. Por ejemplo, para entender las formas de inserción laboral, las estrategias que utilizan los egresados para conseguir trabajo, las redes 
sociales, los mercados primarios y secundarios, los segmentados, las nuevas formas organizativas del trabajo, las demandas de nuevas competencias, la internacionalización de la producción, los desarrollos locales y regionales, la movilidad laboral, la migración, etc. Temas todos relevantes en una época en que se demanda de la educación innovación en sus prácticas e innovación en el conocimiento.

\section{Modelos a explorar}

Lo que puede considerarse una definición de Vinculación reconocida por los coordinadores de carreras técnicas participantes en el foro referido líneas arriba, y del que derivan estas reflexiones, es la que lo considera como el "proceso que establece la relación entre las carreras técnicas del SEMS y las demandas y retos del entorno, a través de una interacción dinámica y continua con las empresas, instituciones, autoridades y sociedad, en busca de un beneficio mutuo".

De las conclusiones generales de este foro, se rescata que hay una demanda evidente y - muy importante - disposición para analizar y buscar flexibilidad a la normatividad respecto de acuerdos a gestionar desde las escuelas. Por otro lado, que desde el eje de gestión de la vinculación pueden desarrollarse estrategias de financiamiento de la vinculación.

Una de las propuestas a las que se llegó fue continuar con espacios de discusión como el que el SEMS abrió con dicho Foro. Inclusive iniciar un Foro Virtual para mantener discusiones tópicas e intercambios de experiencias (que no se materializó, a pesar del interés mostrado por los coordinadores y la relativa facilidad de hacerse por Internet).

Un modelo interesante a explorar es la llamada Interfaz UniversidadEmpresa, promocionada por la Agencia Española de Cooperación Internacional, que periódicamente abre convocatoria para que las Instituciones 
de Enseñanza Superior (IES) desarrollen o mejores sus programas de vinculación con el sector productivo.

Otro, lo constituye lo que se conoce como "formación en la transición” que manejan algunos organismos internacionales como La Fundación Internacional para la Juventud (FIJ) y el Fondo Multilateral de Inversiones (FOMIN) del Banco Interamericano de Desarrollo (BID), con el apoyo de la Agencia de Cooperación del Gobierno de los Estados Unidos (USAID), que manejan el programa - "Entra 2I"- que cofinancia proyectos de empleo juvenil en tecnologías de información (TI).

Por supuesto, y no menos importante por cierto, la participación en redes de investigación aplicada como por ejemplo redEtis (Red de educación, trabajo e inserción social), que a nivel latinoamericano tiene una presencia y autoridad reconocida en personalidades como María de Ibarrola y Eduardo Pieck, por citar a algunos de los muchos con producción muy importante.

\section{Conclusión}

La reforma a la Educación Media Superior es una tarea inaplazable, sobre todo porque la tendencia demográfica indica una mayor demanda al nivel, y en mucho las acciones de planeación educativa tendrán mas que un carácter prospectivo, uno correctivo, si se porterga.

Por otro lado, la necesaria reforma del nivel no es una tarea exclusiva del gobierno ni de las instituciones educativas, debe ser una tarea en la que se incorporen los distintos actores sociales, buscando crear los espacios o aprovechar los ya existentes para alentar la participación social en este nivel educativo. Ello, si es que se pretende que las acciones, las metas y los propósitos tengan una mayor envergadura. 
En la vía de elevar la calidad de la educación superior, el programa sectorial de educación propone como estrategia definir "las competencias para la vida y el trabajo que sean los mínimos requeridos para obtener una certificación nacional de educación media superior". Para ello propone establecer como actividad permanente la vinculación con las comunidades y su ambiente laboral, así como suscribir convenios de colaboración con IES para prácticas, conferencias, talleres, acceso a proyectos de investigación, etc.

En este sentido, el programa sectorial no plantea estrategias novedosas, al menos para el SEMS de la U de G debido en parte por su carácter de bachillerato universitario, que tiene eso sí una oportunidad de potenciar su vinculación con el nivel superior,

No se observan, por supuesto, en estas líneas las particularidades de las diferentes escuelas y las carreras que imparten en el SEMS, pero se pueden desarrollar a partir de la antigüedad de las carreras ofertadas, los tipos de mercados de trabajo con los que en virtud del área de conocimiento se vinculan de manera "natural"; otro criterio de análisis puede ser el carácter metropolitano o regional de las escuelas.

Por otro lado, la evaluación del impacto de las actividades de vinculación de las escuelas del SEMS, preferentemente debe desarrollarse con el apoyo de indicadores, que existen, pero que se han de adecuar a las peculiaridades del nivel educativo.

La vinculación más dinámica, creativa y desprejuiciada con empresas tanto locales como nacionales e internacionales tiene potencialidades que puede ayudar a romper un círculo vicioso... o perverso más bien, en que los sectores educativo y productivo se reclaman responsabilidades incumplidas.

Qué tanto pueda romperse este círculo a partir de la reforma del nivel medio superior es algo que puede ya plantearse como una situación a indagar a la luz de las acciones implicadas en la Reforma Integral de la 
Educación Media Superior que ha impulsado a nivel federal la Subsecretaría de Educación Media Superior, que empezó a caminar en 2009 con un programa nacional de formación docente para favorecer la educación basado en competencias. Es el mismo caso de la Universidad de Guadalajara, que prácticamente a la par de la reforma federal, ha iniciado una reforma curricular del bachillerato. A ambos niveles, resultan una veta investigativa sumamente interesante.

\section{THE BACHELOR'S AND THE REFORM OF UPPER-MIDDLE, THE POTENTIAL FOR LINKING}

The creation of a SubMinistry for High School Education, besides could be regarded as a sign of the importance that the government wants assign to this level, offers possibilities to link more productively the actions of public policy with the actions of the educational institutions, and they, at its turn, with the actions that need to be implemented, concerted at the level of whole society, to achieve progress in the country. Apparently there is a will and determination of all social sectors to devote increasing resources to the level. There is a national reform of the baccalaureate, which is guided by a competency model, a situation which, the baccalaureate of the University of Guadalajara can not be on the sidelines. This work is a reflection on the search for identity for the high school linkage of the University of Guadalajara, which offers general and technical baccalaureate as well as technical careers. The activity from which it reflects was directed to rescue experiences of different schools that integrates the Sistema de Educación Media Superior and search empower toward ways of innovative linkage.

KEY WORDS: Higher education average. Linkage. Reforms of the baccalaureate. 


\section{Referencías}

AGENCIA ESPAÑOLA DE COOPERACIÓN INTERNACIONAL. Disponível em: <www.aecid.es/>. Acesso em: 20 fev. 2007.

FUNDACIÓN INTERNACIONAL PARA LA JUVENTUD. Disponível em: <http:// www.iyfnet.org/>. [12/02/o8]

OBSERVATORIO CIUDADANO DE LA EDUCACIÓN. Nivel medio superior ¿eslabón perdido de la educación? Comunicado I9. Disponível em: <http://www.observatorio.org/>. Acesso em: 5 fev. 2008.

ORGANISATION FOR ECONOMIC CO-OPERATION AND DEVELOPMENT (OCDE). Education at a glance. Disponível em: <http://www.oecd.org/ dataoecd/36/4/4070I218.pdf>.

PLAN NACIONAL DE DESARROLLO 2007-2012. Disponível em: <www.pnd. presidencia.gob.mx>.

PROGRAMA SECTORIAL DE EDUCACIÓN 2007-20I2. Disponível em: < http://www. sep.gob.mx/work/sites/sepi/archivos/prog_sec.pdf>.

RED DE EDUCACIÓN, TRABAJO E INSERCIÓN SOCIAL. Disponível em: <http:// www.redetis.iipe-ides.org.ar $>$. Acesso em: 20 fev. 2008.

RODRÍGUEZ, ROBERTO. El bachillerato, cirugía mayor. Disponível em: <http:// rodriguez.blogsome.com/campus-I 87/>. Acesso em: 3 dez. 2007.

SEP. (2004) Sistema educativo de los Estados Unidos Mexicanos - Principales cifras, ciclo escolar, 2003-2004. Disponível em: <http://cenedic.ucol.mx/bibliosep/recursos/294.pdf>. Acesso em: 20 maio 2008.

Recebido em 7 abr. 2010 / Aprovado em 9 dez. 2010

\section{Para referenciar este texto}

RUIZ FLORES, J. El bachillerato universitario y la reforma del nivel medio superior, potencialidades para la vinculación. EccoS, São Paulo, v. I2, n. 2, p. 423-435, jul./dez. 2010. 
